Supporting Information for

\title{
Superhydrophobic Covalent Organic Frameworks Prepared via Pore Surface Modifications for Functional Coatings under Harsh
}

\section{Conditions}

Na Han* 1,2,3, Zongxuan Zhang ${ }^{1,2}$, Hongkun Gao ${ }^{1,2}$, Yongqiang Qian ${ }^{1,2}$, Linli Tan ${ }^{1,2}$, Chao Yang ${ }^{1,2}$, Haoran Zhang ${ }^{1,2}$, Zhenyu Cui1 ${ }^{1,2}$, Wei Li ${ }^{1,2}$, Xingxiang Zhang* ${ }^{1,2}$

${ }^{1}$ State Key Laboratory of Separation Membranes and Membrane Processes, Tiangong University, Tianjin 300387, China;

${ }^{2}$ School of Materials Science and Engineering, Tiangong University, Tianjin 300387, China;

${ }^{3}$ Department of Textile Engineering, Chemistry \& Science, College of Textiles, North Carolina State University, Raleigh, NC, 27606, United States.

* Corresponding author:

E-mail address: nhan@ncsu.edu; zhangtjpu@ hotmail.com

The crystalline structure of COF-DhaTab was confirmed by PXRD analysis as shown in Figure Aa. Unit cell models of AA-stacking and AB stacking are shown in Figure Ab and c. In the experimental PXRD profile of COF-DhaTab, a strong peak at $2.73^{\circ}$, and other comparatively weaker peaks at $4.8,5.6,7.4,9.6$, and $26.3^{\circ}$ were observed, which was consistent with the simulation results obtained with Materials studio (Figure $2 \mathrm{~b}$ and c). Figure B1 shows the Fourier transform infrared (FT-IR) spectrum of COF- 
DhaTab and raw materials. Compared to raw materials, the appearance of the stretching band for $\mathrm{C}=\mathrm{N}$ at $1612 \mathrm{~cm}^{-1}$ was observed, and monomer characteristic peaks of $\mathrm{C}=\mathrm{O}$ at $1668 \mathrm{~cm}^{-1}$ and N-H at $\sim 3300 \mathrm{~cm}^{-1}$ disappeared.

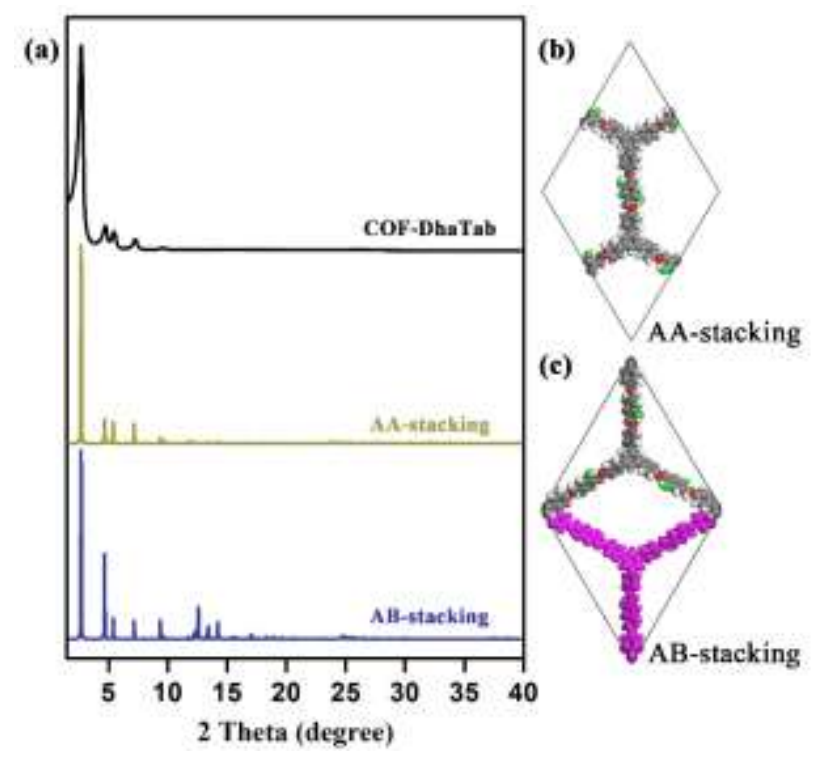

Figure A. (a) PXRD profiles of experimental results (black), simulated results using AA-stacking (purple) and AB-stacking (green) of COF-DhaTab. The unit cell of (b) AA-stacking and (c) AB-stacking.

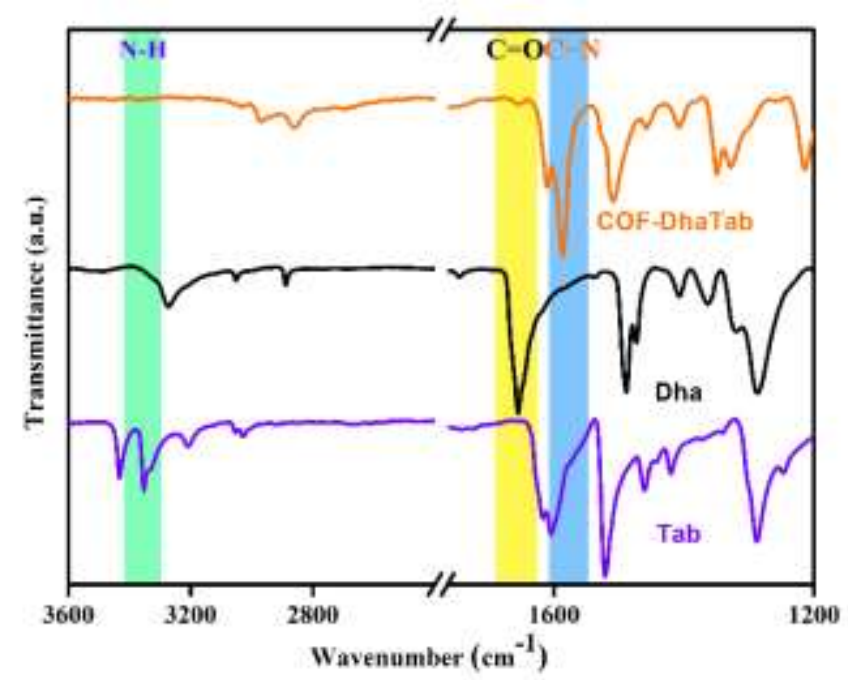

Figure B. The Fourier transform infrared spectrum of COF-DhaTab and monomer. 


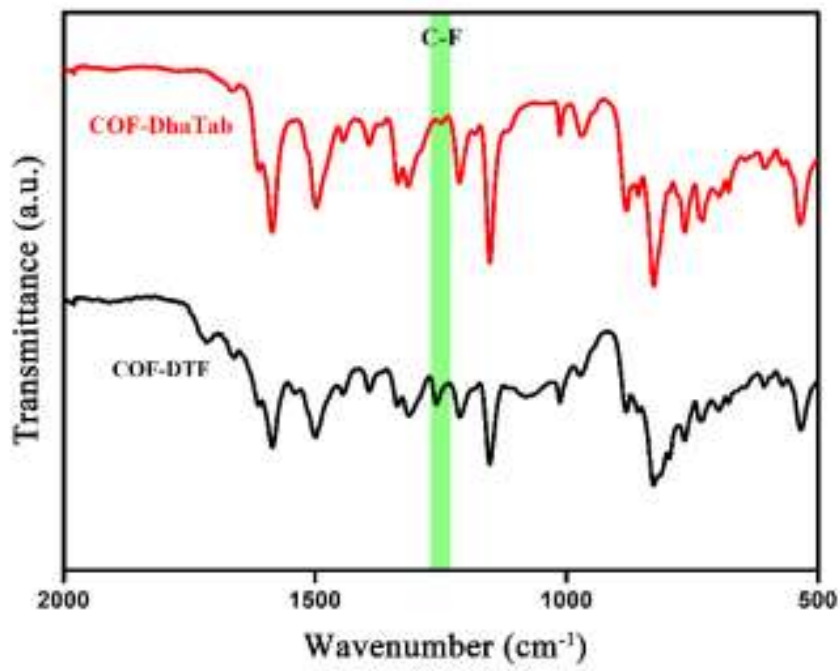

Figure S1. FT-IR spectra of COF-DhaTab and COF-DTF.
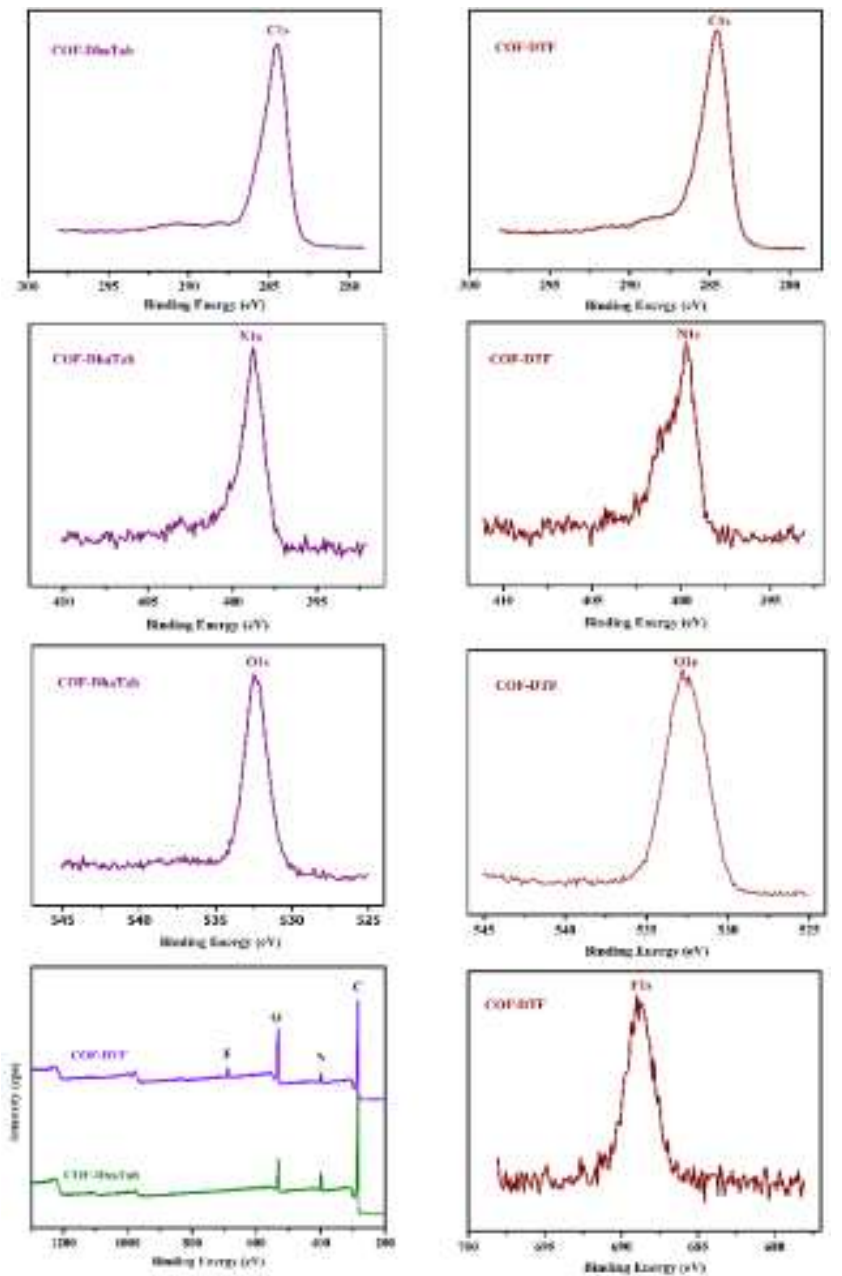
Figure S2. XPS spectra of COF-DhaTab and COF-DTF.
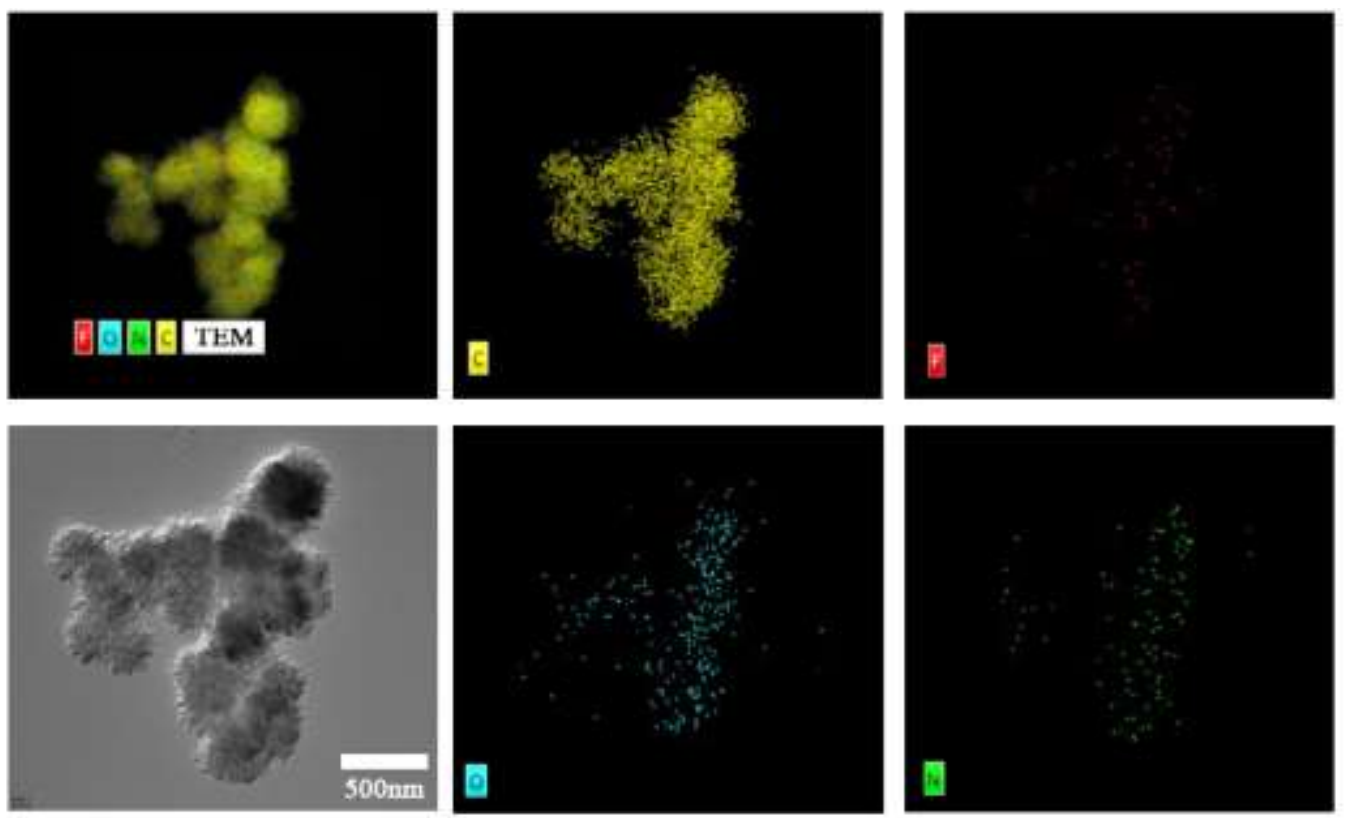

Figure S3. TEM and corresponding element mapping of COF-DTF.
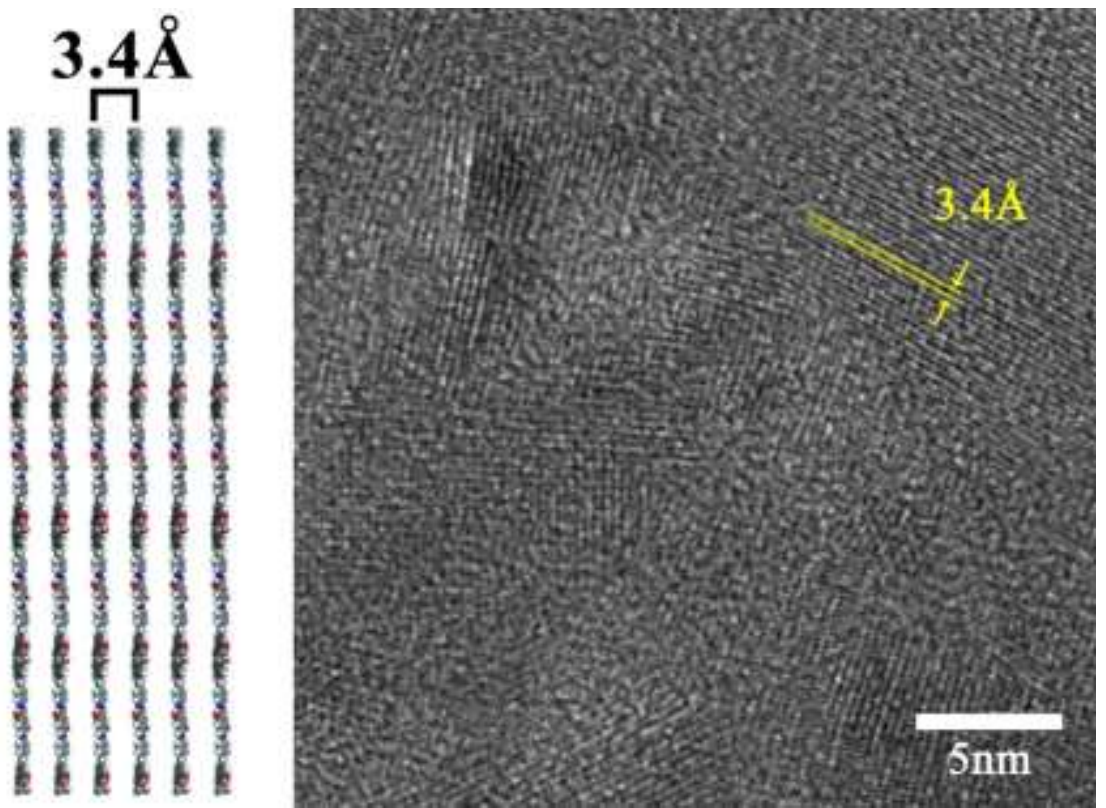

Figure S4. HRTEM image of COF-DTF, presence of lattice fringes indicating its high degree of crystallinity and the distance between the adjacent fringes is consistent with the interlayer spacing of the proposed structure. 


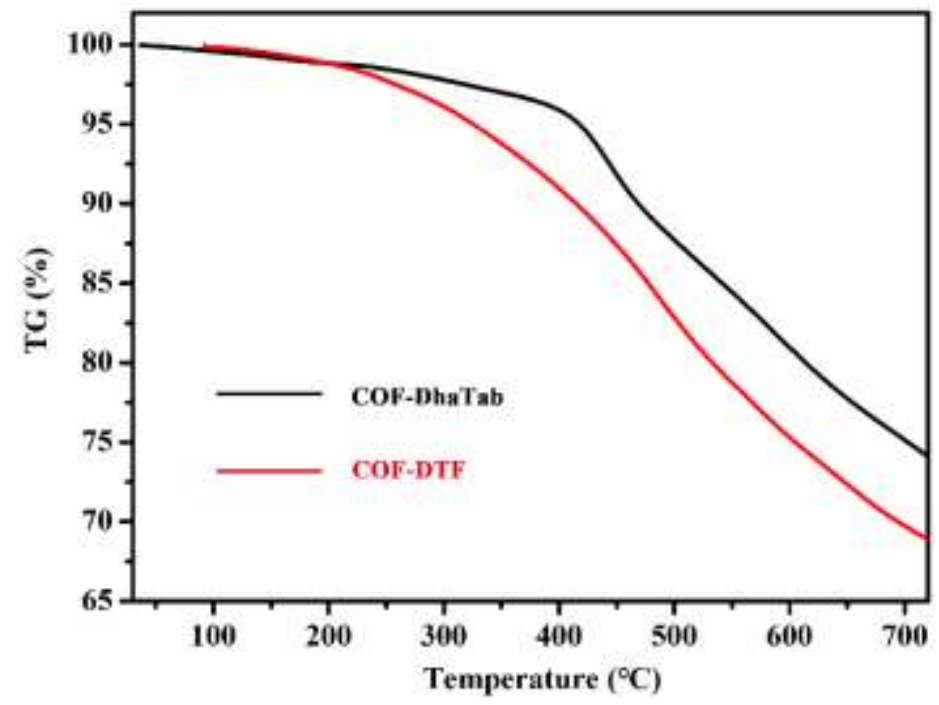

Figure S5. TG curves of COF-DhaTab and COF-DTF tested under $\mathrm{N}_{2}$ atmosphere.

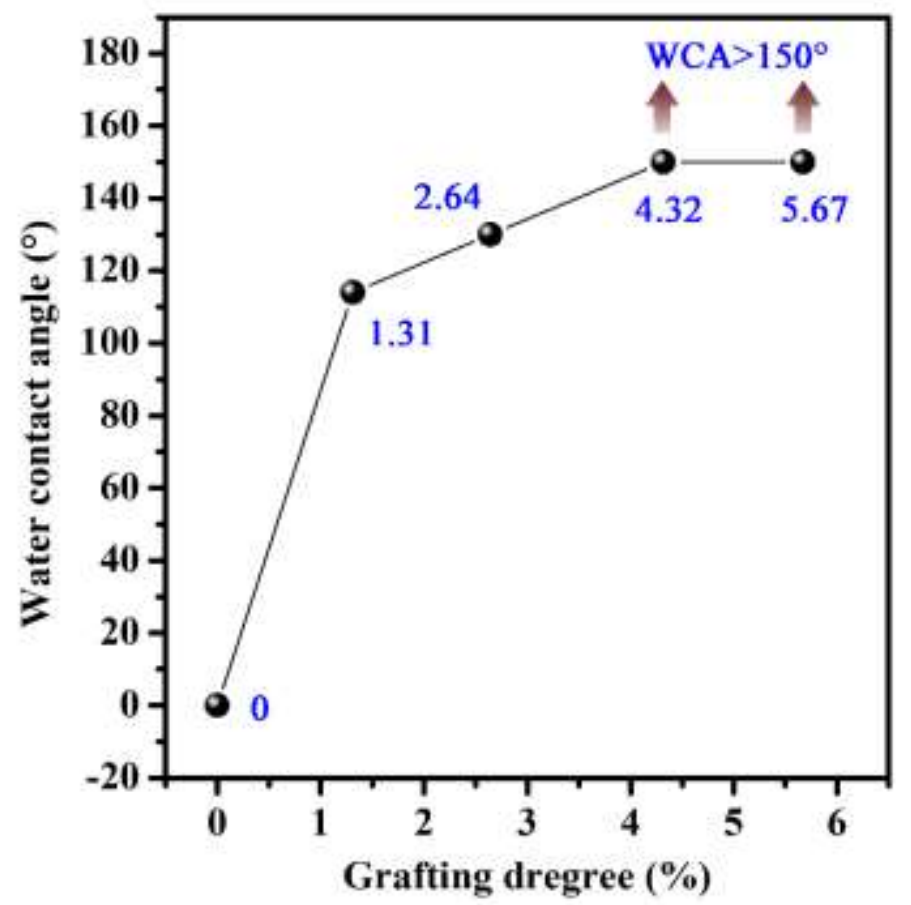

Figure S6. The relationships between water contact angle and grafting degree (fluoride) of COF-DTF. 


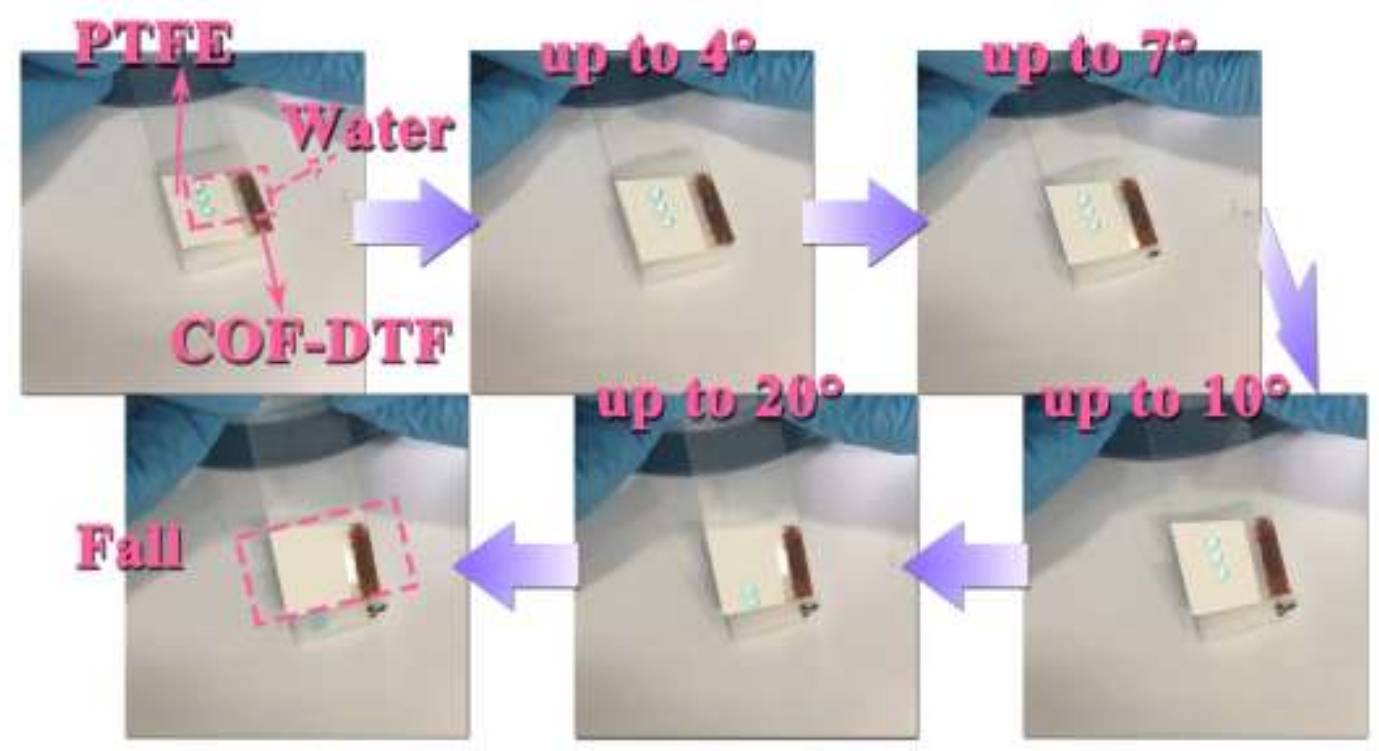

Figure S7. The water droplets slide contrast photograph of COF-DTF coating and PTFE membrane.

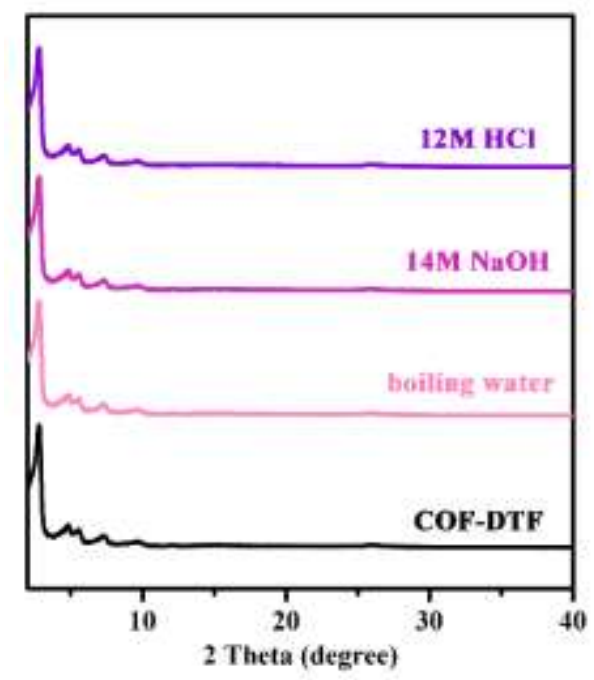

Figure S8. PXRD patterns of COF-DTF treated in various harsh conditions for 7 days. 


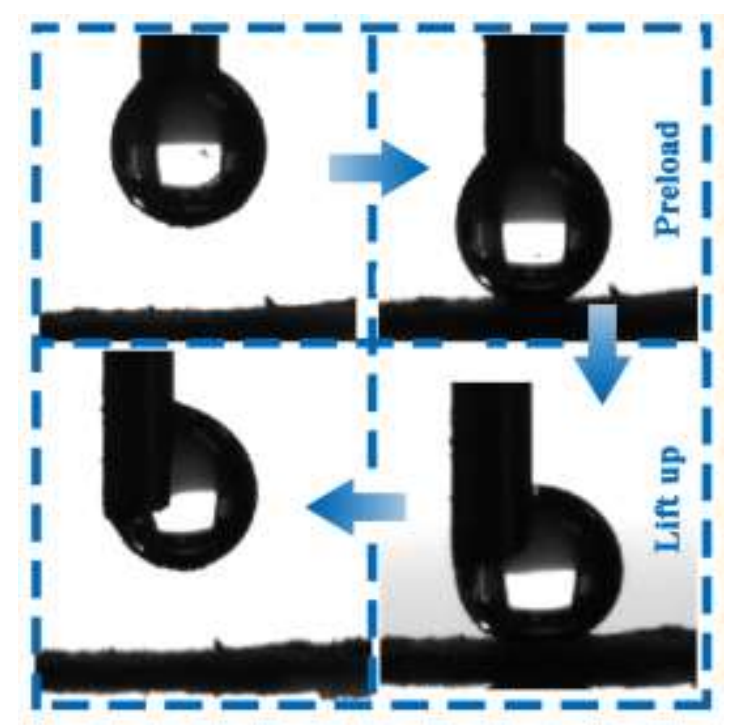

Figure S9. The dynamic water adhesion test of COF-DTF after 1 week of being treated in $\mathrm{HCl}(12 \mathrm{M})$.

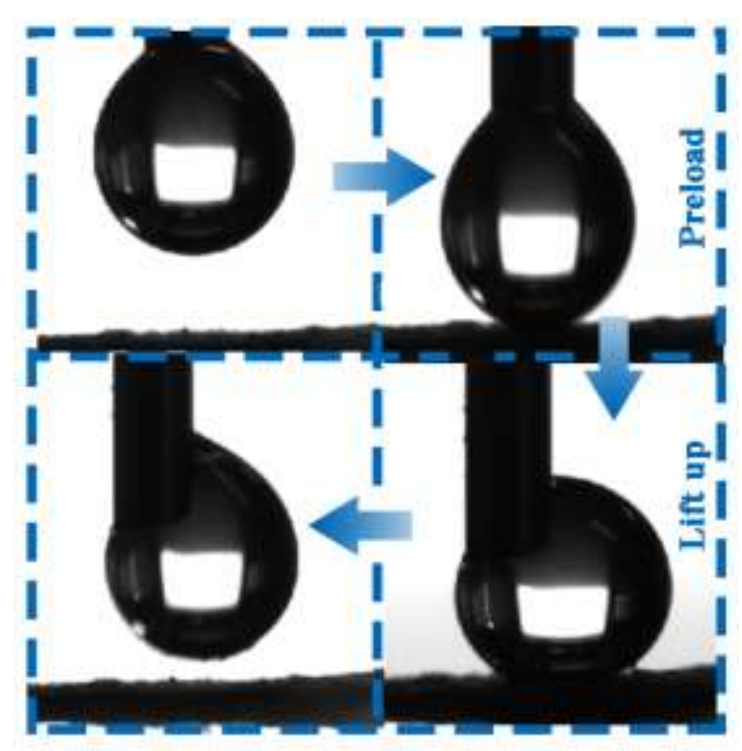

Figure S10. The dynamic water adhesion test of COF-DTF after 1 week of being treated in $\mathrm{NaOH}(14 \mathrm{M})$. 


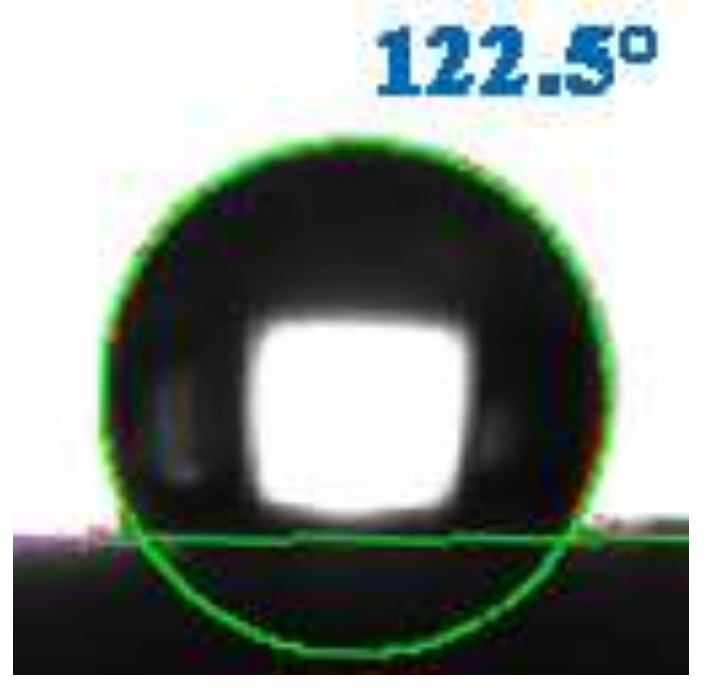

Figure S11. The water contact angle of COF-DhaTab\&F.

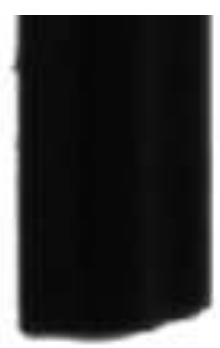

\section{s옹줄}

In acetone

Figure S12. The water contact angle of COF-DhaTab\&F (after soaking in acetone). 

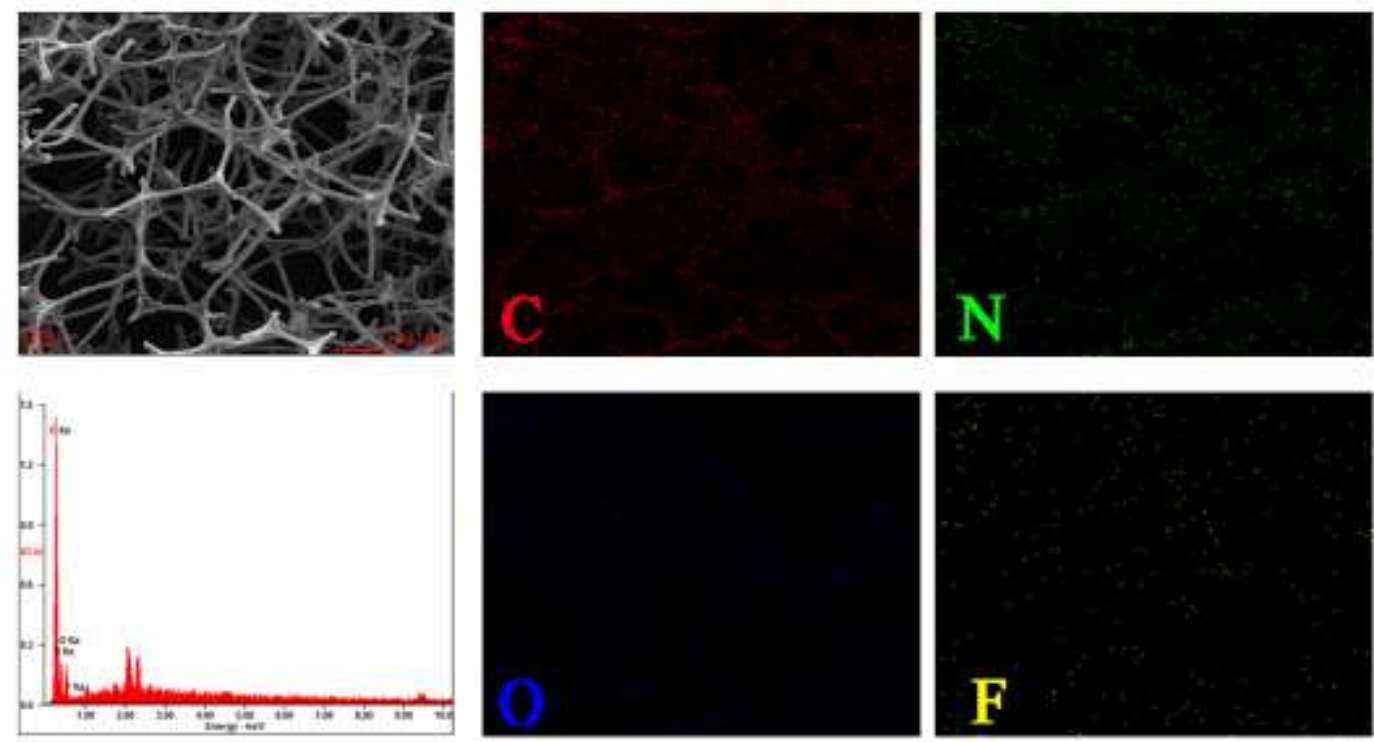

Figure S13. SEM and corresponding element mapping of COF-DTF@foam.

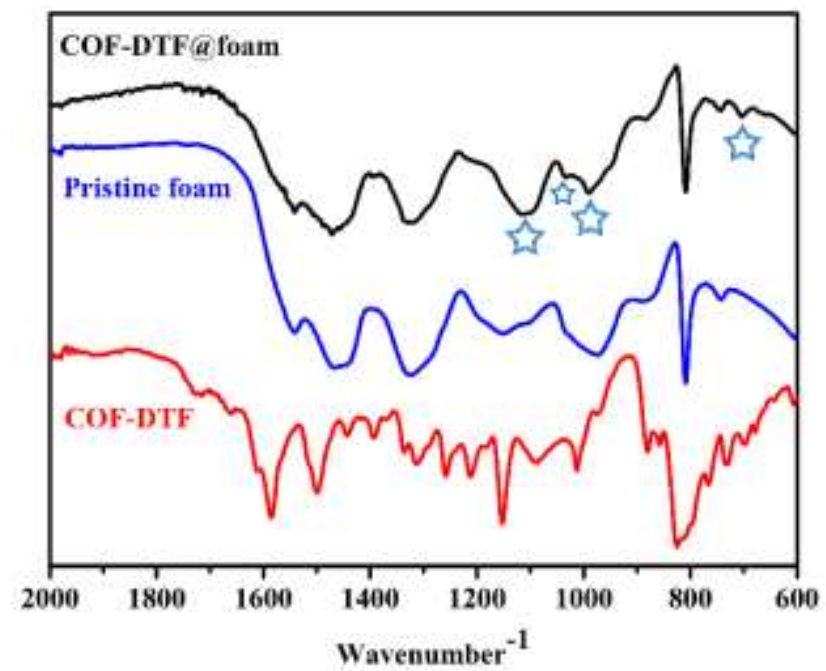

Figure S14. FTIR spectra of COF-DTF, pristine foam, and COF-DTF@foam.

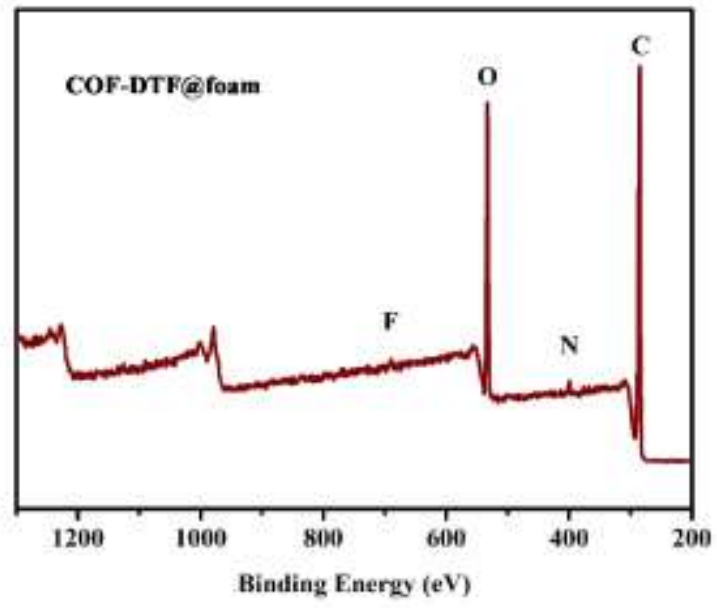


Figure S15. XPS spectra of COF-DTF@foam.

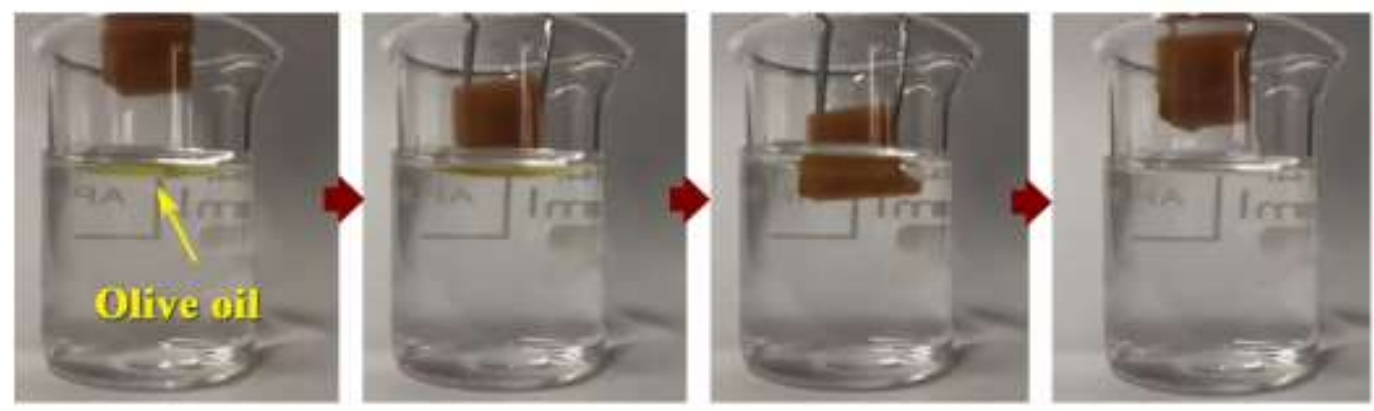

Figure S16. The photographs of adsorption process of light oil (olive oil) by COF-

\section{DTF@foam}

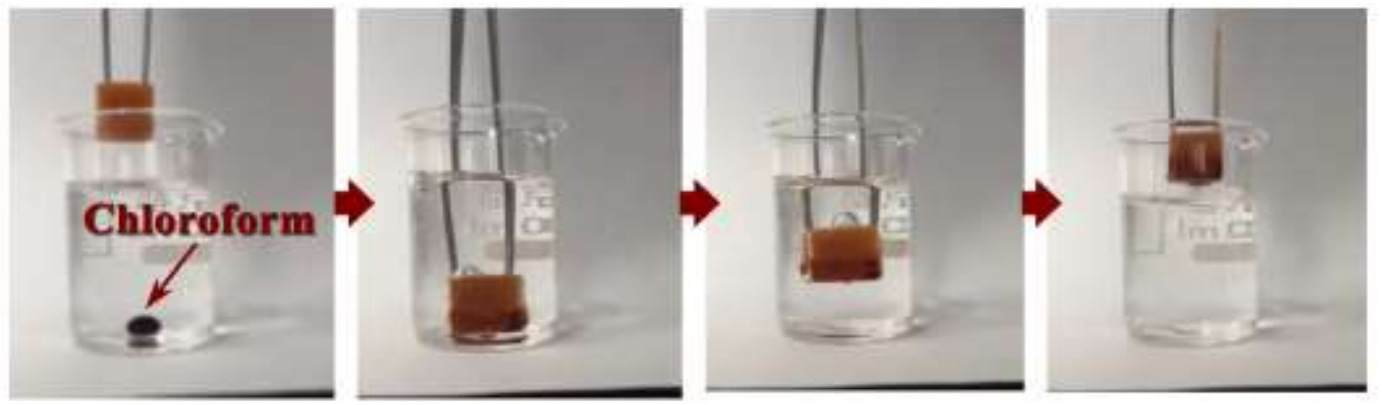

Figure S17. The photographs of adsorption process of heavy oil (chloroform) by COF-

DTF@foam.

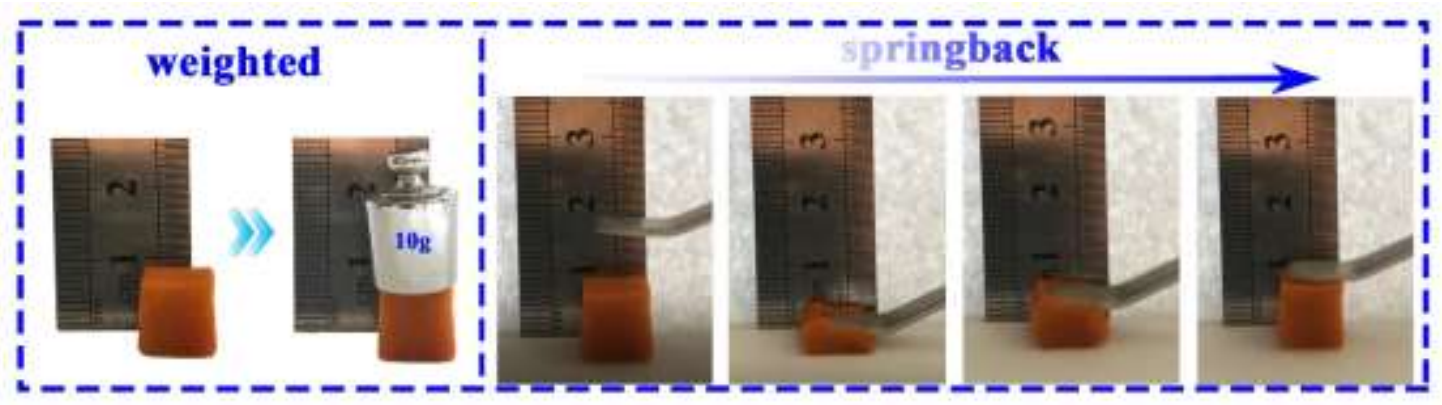

Figure S18. The image of weight-bearing and springback of COF-DTF@foam. 

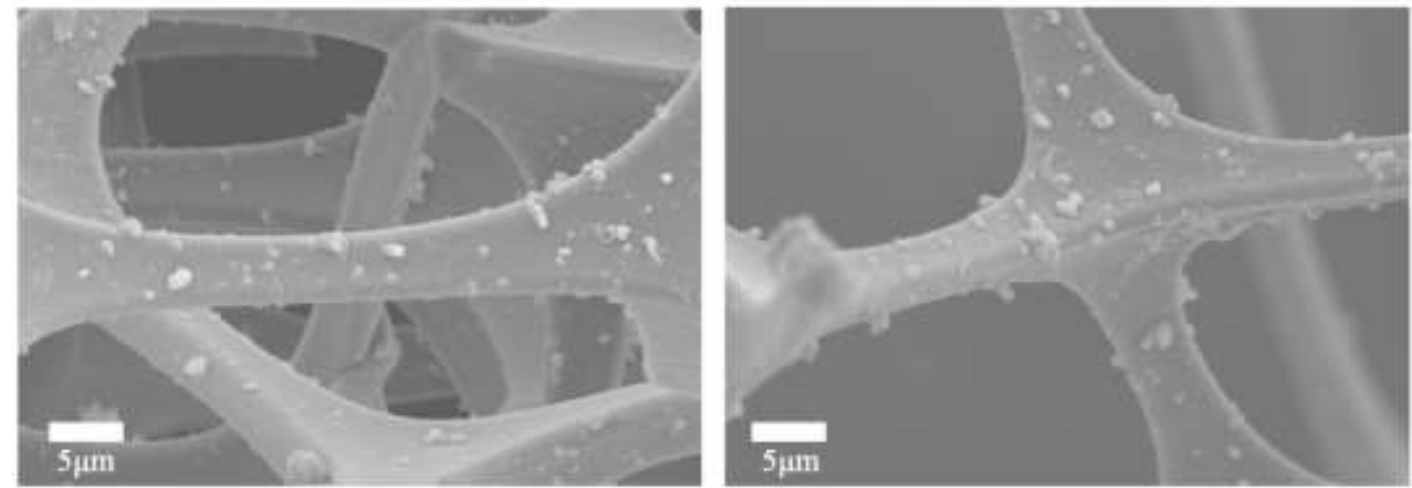

Figure S19. SEM image of COF-DTF@foam before and after 10 rounds of adsorption/squeezing.

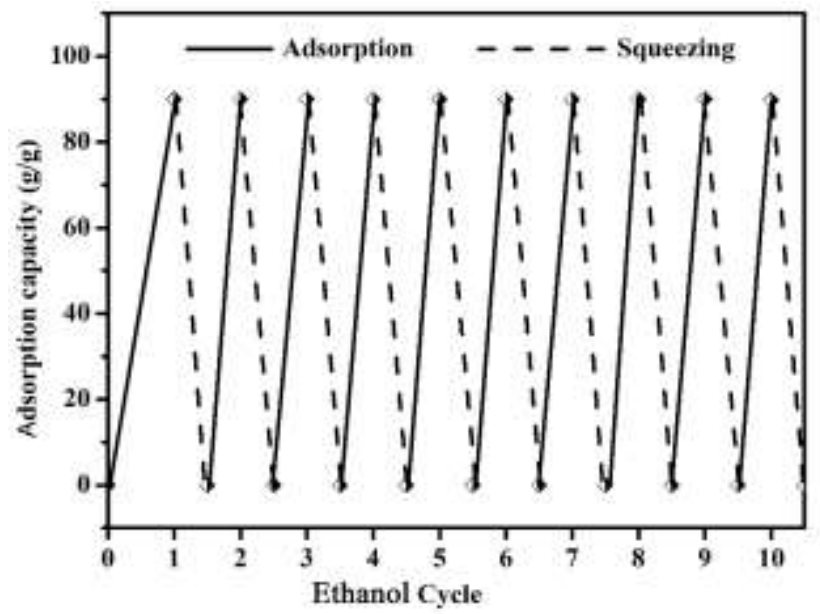

Figure S20. The recycling adsorption performance for ethanol of COF-DTF@foam was extruded 10 times.

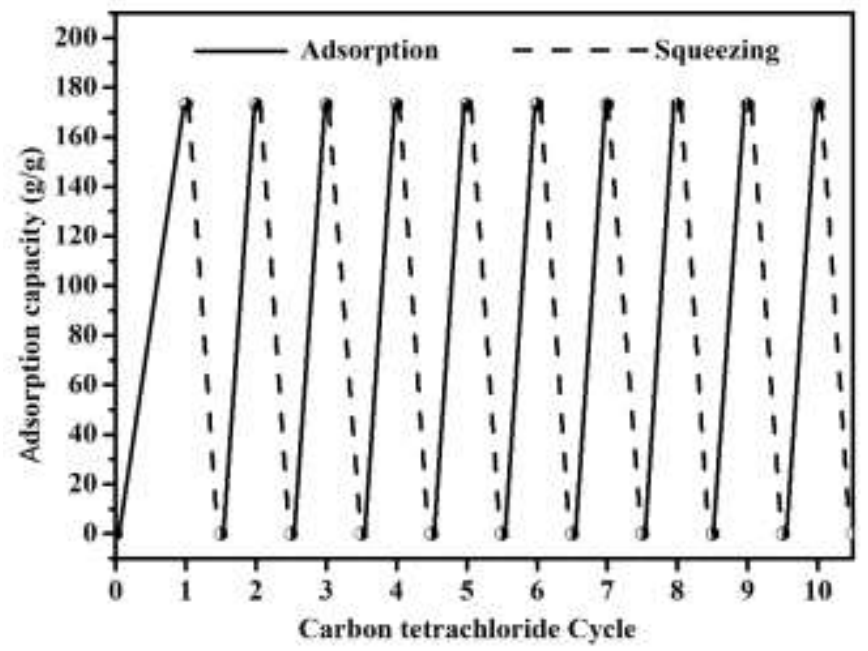

Figure S21. The recycling adsorption performance for carbon tetrachloride of COF- 
DTF@foam was extruded 10 times.

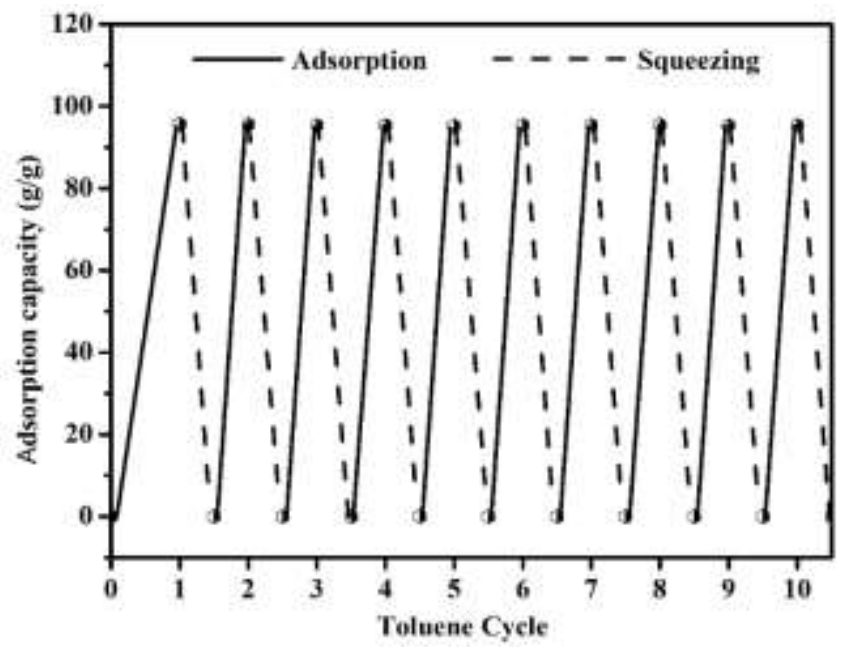

Figure S22. The recycling adsorption performance for toluene of COF-DTF@foam was extruded 10 times.

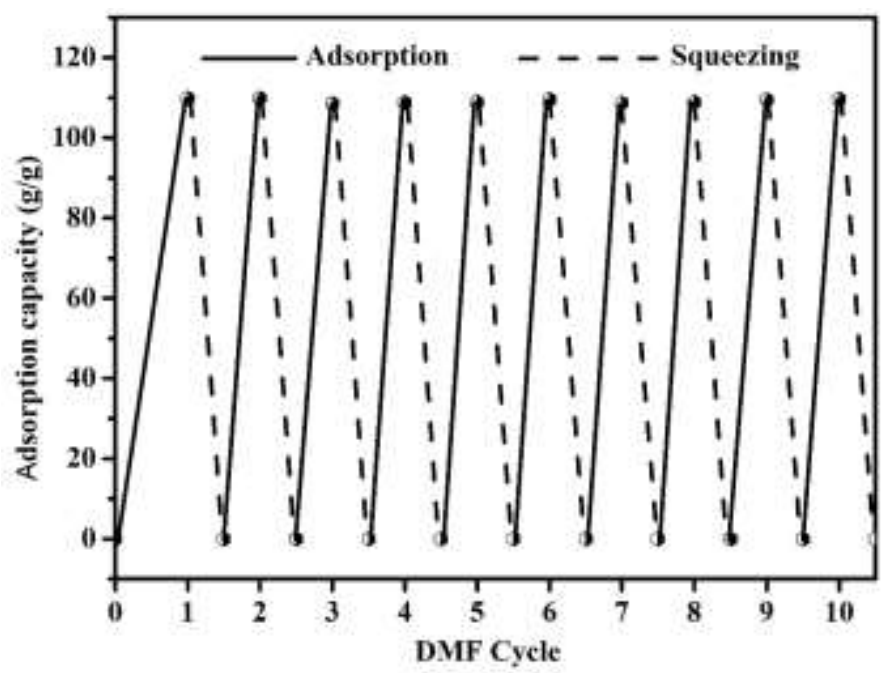

Figure S23. The recycling adsorption performance for DMF of COF-DTF@foam was extruded 10 times. 


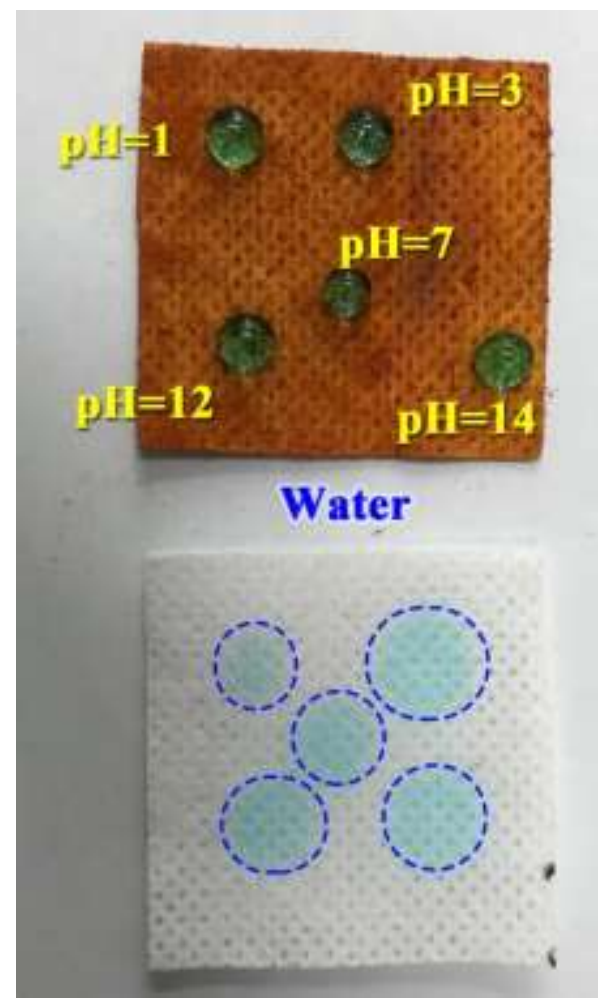

Figure S24. The water droplet status of different $\mathrm{pH}$ on the surface of pristine cloth and COF-DTF@cloth.

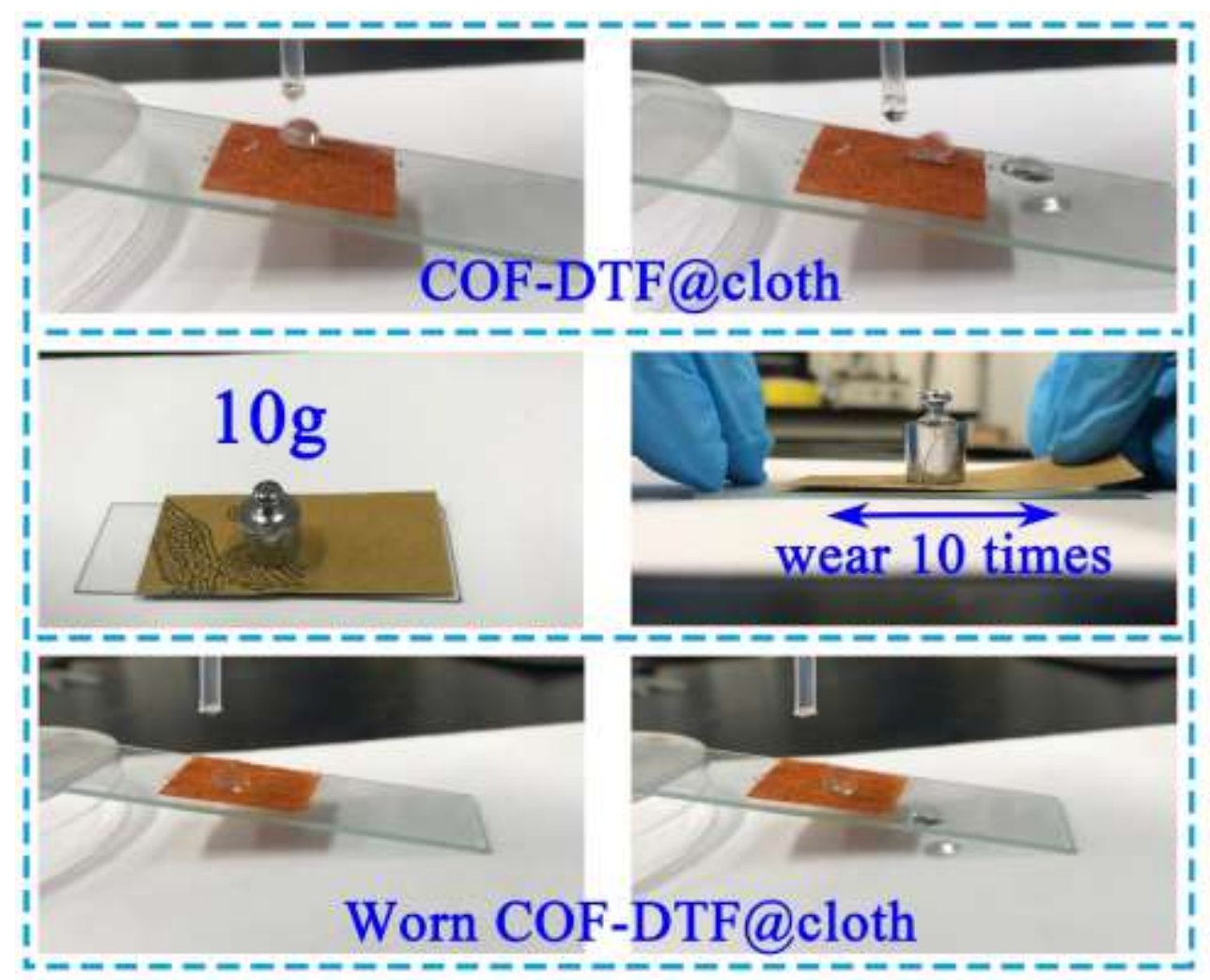

Figure S25. The self-healing ability test of COF-DTF@cloth. 